\title{
Research on the effects of family care degree on stigma and psychosocial adaptation among the patients with Parkinson's disease ${ }^{\dagger}$
}

Original article

Yue-Yue He $\mathrm{He}^{\mathrm{a}}$, An-Chun Yin ${ }^{\mathrm{b}, *}$

${ }^{a}$ Dalian Medical University, Dalian, Liaoning 116044, China

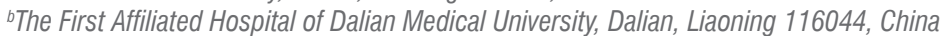

Received: 20 April 2020; Accepted: 22 June 2020; Published: 20 March 2021

Abstract: Objective: This study aimed to investigate the status quo of family care degree, stigma and psychosocial adaptation of the patients with Parkinson's disease (PD), and discuss the effects of family care degree on stigma and psychosocial adaptation among the patients with PD.

Methods: This study was conducted between September 2018 and April 2019 on patients with PD who were admitted to a Tertiary Grade A hospital in Dalian city. A total of 246 PD patients were investigated by convenience sampling method using self-designed general information questionnaire, along with family care index (APGAR), 24-item Stigma Scale for Chronic Illness (SSCI), and Psychosocial Adjustment Scale (PAS) for PD. And patients were divided into two groups according to the level of family care degree and compared with stigma and psychosocial adaptation.

Results: The total score of family care for patients with PD was $6.97 \pm 2.46$, family function was good in 132 cases $(53.7 \%)$, moderate barrier in 92 cases (37.4\%), and severe barrier in 22 cases (8.9\%). The scores of stigma and psychosocial adaptability and their dimensions in the group with high degree family care were higher than the low degree group.

Conclusions: The overall level of family care for PD patients was poor. Improving family care degree can help patients with PD to reduce stigma and improve psychosocial adaptation.

Keywords: Parkinson's disease • family care • stigma • psychosocial adaptability • quality of life

(c) Shanxi Medical Periodical Press.

\section{Introduction}

Parkinson's disease (PD) is a common degenerative disease of the central nervous system in the elderly, which is the second largest neurodegenerative disease after senile dementia. ${ }^{1,2}$ There is no effective way to slow or stop the progression of the PD that is mainly treated by drugs and surgery. ${ }^{3}$ In recent years, more and

† This project was supported by Liaoning Economic and Social Development Project (No. 2015Islktzijjx-13) and Technical Project of Science and Technology Department of Liaoning Province (No. 2013225002).

How to cite this article: He YY, Yin AC. Research on the effects of family care degree on stigma and psychosocial adaptation among the patients with Parkinson's disease. Front Nurs. 2021;1:69-74.

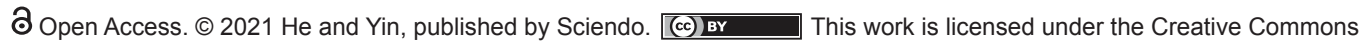
Attribution 4.0 License. 
more experts at home and abroad pay greater attention to the quality of life of patients with PD. ${ }^{4,5}$ In a prospective cohort study, Ma et al. ${ }^{6}$ argued that stigma is a key determinant of quality of life of patients with PD, and medical staff should be aware about the stigma associated with PD. Meanwhile, Zhang et al. ${ }^{7}$ believed that psychosocial adaptation of PD is also an important factor affecting the quality of life. This paper considers that the subjective attitude of family function on patients with PD may change and have an impact on stigma and psychosocial adaptation. Furthermore, this study not only discusses how family functionality can affect the stigma and psychosocial adaptation in the context of Chinese but also provides theoretical basis for the study of family function and improving quality of life of patients with PD, and the report is as follows.

\section{Subjects and Methods}

\subsection{Subjects of the study}

A total of 246 patients with PD between October 2018 and April 2019 were selected by the convenient sampling method who met the inclusion and exclusion criteria.

Inclusion criteria are as follows: diagnosed with PD and the diagnostic standard is "China's diagnostic standard for PD (2016)"”; >18 years old; informed consent; and willingness to participate in this study. Exclusion criteria are as follows: serious diseases of other systems, such as liver and kidney failure, advanced cancer and the like; a history of mental illness; serious communication difficulties; and cannot cooperate.

\subsection{Research tool}

\subsubsection{General information questionnaire}

The questionnaire was designed by the researchers themselves according to the purpose of the study, including age, sex, medical care payment mode, education level, marital status, living status, etc.

\subsubsection{Family APGAR index}

The family care degree was evaluated by Family APGAR index ${ }^{9}$ for Chinese samples. ${ }^{10}$ This tool aimed to identify signs of family dysfunction and it is also called the family function assessment scale. The five dimensions of family care degree are adaptability, partnership, growth, affection, and resolve. For each one, respondents could choose a value from an ordinal 3-point scale: 0 (hardly ever), 1 (some of the time), and 2 (almost always). For the statistical analysis, family function scores were used as a continuous variable, as well as categorized as follows: high family dysfunction $=0-4$ points, moderate family dysfunction $=5-6$ points, and preserved family dysfunction $=7-10$ points. Final scores ranged from 0 to 10 . The higher the score, the better the family function and higher is the individual perception toward the relation to family care degree.

\subsubsection{4-item Stigma Scale for Chronic IIIness}

The Chinese Version of Stigma Scale for Chronic IIIness (CSSCl) was used to assess the stigma. The compilations by Deng et al. ${ }^{11}$ was used to measure stigma in patients with chronic neurological diseases. It is composed of 24 questions, and responses regarding the frequency of occurrences (FO) were arranged on an ordinal 5-point scale: $1=$ Never, 2 = Rarely, 3 = Sometimes, 4 = Often, and 5 = Always. The raw summed score range for the Stigma Scale for Chronic Illness (SSCI) accordingly was 24-120. High scores indicated greater individual experience of stigma. In 2017, Deng et al. ${ }^{11}$ developed a CSSCI and tested its total reliability and validity of scale on the patients with cerebral apoplexy. Its Cronbach's a coefficient was 0.951 and retest reliability was 0.881 , which proves that CSSCI has good reliability and validity of entries straightforward. And also CSSCI has good applicability because the process could be completed easily in 6-10 $\mathrm{min}$.

\subsubsection{Psychosocial Adaptation Scale for $P D$}

To measure Psychosocial Adaptation, we used the Psychosocial Adjustment Scale (PAS) for PD compiled by Zhang et al. ${ }^{7}$ PAS incorporated 6 dimensions: anxiety, self-esteem, attitude, self-acceptance, self-efficacy, and social support, a total of 32 items. The test-retest correlation coefficient was 0.99 and average content validation rate was 0.97 .

\subsection{Research method}

This study was conducted between September 2018 and April 2019 on patients with PD, who were admitted to Three-A hospital in Dalian city. The method of questionnaire was used for investigation in this study; these were unified questionnaires which ought to be filled truthfully. The patients were asked to fill out the questionnaire according to the patient's perception of the true answer. If the patient was not able to fill himself due to reasons such as rest tremor, the researcher or his family members would request patient to answer of each item one by one. At the day of the recovery, a total of 260 questionnaires were distributed; of those, 246 
questionnaires were recovered at an effective recovery rate of $94.6 \%$.

\subsection{Statistical method}

Once all the data were collected, a database was set up. The analysis of database was performed by SPSS24.0 software (IBM Corporation, Armonk, New York, United States), including descriptive statistics analysis and independent sample $t$-test, which were used to explore the influence of family care on stigma and psychosocial adaptation. The independent sample $t$-test was applied to compare the stigma and psychosocial adaptation of PD patients with different levels of family care.

\subsection{Ethical principles}

The study was approved by the Research Ethics Committee of The First Affiliated Hospital of Dalian Medical University, and all participants signed a statement of informed consent.

\section{Results}

\subsection{Sociodemographic profile of the sample}

Variables were participants from Chronic disease insurance $(56.5 \%)$, male (51.6\%), 60-69 years old $(37.4 \%)$, junior high school or below $(48.4 \%)$, married $(81.3 \%)$, living with his/her spouse $(81.7 \%)$, personal monthly income $<3000$ yuan, and time of illness 1-3 years (37.8\%) (Table 1).

\subsection{Descriptive statistics of family care degree}

Regarding family care degree, the total score of family care for patients with PD was $6.97 \pm 2.46$. The categorical data for this measure showed that the total score of family care for patients with PD was $6.97 \pm 2.46$, family function was good in 132 cases $(53.7 \%)$, moderate barrier in 92 cases (37.4\%), and severe barrier in 22 cases $(8.9 \%)$. About half of the patients had well-functioning families.

\subsection{Independent sample $t$-test of stigma in different family dysfunction groups}

The independent sample $t$-test showed that participants who reported high levels of family function scored lower on stigma $(51.87 \pm 10.54)$ than those who reported low levels of family function $(59.11 \pm 10.70)$, and this difference was statistically significant $(P<0.001)$ (Table 2$)$.

\begin{tabular}{|c|c|c|}
\hline Sociodemographic variables & $\begin{array}{l}\text { Number of } \\
\text { people }(n)\end{array}$ & $\begin{array}{l}\text { Constituent } \\
\text { ratio (\%) }\end{array}$ \\
\hline \multicolumn{3}{|l|}{ Medical care payment mode } \\
\hline Self-paying & 31 & 12.6 \\
\hline Chronic disease insurance & 139 & 56.5 \\
\hline Other insurance & 76 & 30.9 \\
\hline \multicolumn{3}{|l|}{ Sex } \\
\hline Male & 127 & 51.6 \\
\hline Female & 119 & 48.4 \\
\hline \multicolumn{3}{|l|}{ Age (years) } \\
\hline$<60$ & 55 & 22.3 \\
\hline $60-69$ & 92 & 37.4 \\
\hline 70-79 & 79 & 32.1 \\
\hline$\geq 80$ & 20 & 8.1 \\
\hline \multicolumn{3}{|l|}{ Education } \\
\hline Junior high school or below & 119 & 48.4 \\
\hline $\begin{array}{l}\text { High school or technical } \\
\text { secondary school }\end{array}$ & 69 & 28.0 \\
\hline Junior college & 46 & 18.7 \\
\hline Undergraduate or above & 12 & 4.9 \\
\hline \multicolumn{3}{|l|}{ Marital status } \\
\hline Single & 6 & 2.4 \\
\hline Married & 200 & 81.3 \\
\hline Widowed/divorced/separated & 40 & 16.3 \\
\hline \multicolumn{3}{|l|}{ Living status } \\
\hline Living alone & 13 & 5.3 \\
\hline Living with his/her spouse & 201 & 81.7 \\
\hline Living with other members & 32 & 13.0 \\
\hline \multicolumn{3}{|l|}{ Personal monthly income (yuan) } \\
\hline$<3000$ & 115 & 46.7 \\
\hline $3000-5000$ & 94 & 38.2 \\
\hline$>5000$ & 37 & 15.0 \\
\hline \multicolumn{3}{|l|}{ Time of illness (years) } \\
\hline$<1$ & 25 & 10.2 \\
\hline$<3$ & 93 & 37.8 \\
\hline $3-5$ & 59 & 24.0 \\
\hline$>5$ & 69 & 28.0 \\
\hline
\end{tabular}

Table 1. Sociodemographic profile of patients with Parkinson's disease sample studied.

\subsection{Independent sample $t$-test of psychosocial adaptation in different family dysfunction groups}

The independent sample $t$-test showed that participants who reported high levels of family function scored higher on psychosocial adaptation (90.28 \pm 13.50) than those who reported low levels of family function (77.30 \pm 13.70$)$, and this difference was statistically significant $(P<0.001)$ (Table 3$)$. 


\section{Discussion}

\subsection{The status of family care in patients with PD}

The family care degree, considered as a psychological index, is the subjective attitude of patients toward family, which can reflect the true satisfaction of patients with family functions. ${ }^{12}$ The results of this survey showed that the score of family care of PD patients was at the middle level $(6.97 \pm 2.46)$, and about $46.3 \%$ of the patients had family dysfunction. This reveals that the level of family care in patients with PD is not ideal, which is almost consistent with the domestic research results related to this study. ${ }^{13,14}$ The reason may be that the burden and implication of PD is very high and it gets worse and worse as it progresses, which bring great psychological pressure to patients and their families. ${ }^{15}$ Therefore, this study found that the subjective sense of family happiness of PD patients was relatively decreased. It suggests that medical staff should pay greater attention to the impact of changes of family functions on patients while helping patients to treat physical diseases, and encourage patients to actively communicate with their families and express their inner thoughts so that the level of family care degree can be improved.

\subsection{Effect of family care on stigma on PD patients}

The stigma of PD is a feeling of shame and isolation. ${ }^{16}$ It results in decreased social participation, alienation from others or excessive attention to one's own abnormalities caused by symptoms of patients with PD such as changes in body profile or structure, gradual loss of function, and language problems. ${ }^{17}$ Social harmony and keeping them informed regularly are the key to elderly people, and so for elderly, the most important affective relations occur at the family level. ${ }^{18}$ Sun et al. ${ }^{19}$ found that HIVIAIDS patients and its families live in a social environment of stigma and the perceived stigma was related to their family APGAR (OR $=0.650, P=0.027$ ). This study showed that participants who reported high levels of family function scored lower on stigma than those who reported low levels of family function, and this difference was statistically significant $(P<0.001)$. Such results are in line with the majority of the outcomes given in the literature on the subject, which clearly establish that better family function plays an important role in improving stigma of PD. ${ }^{20}$ Patients with PD are often accompanied by complications and side effects of drugs. They require not only financial support from their families to guarantee the use of drugs but also the understanding and companionship of family members to help them in increasing their confidence in treatment and to reduce the pain caused by diseases. Therefore, improving family functions and increasing family members' care are the effective measures to help patients to face stigma correctly. It is worthy of reference for medical staff.

\subsection{Effect of family care on psychosocial adaptation in PD patients}

Psychosocial adaptation of PD refers to the degree to which individuals are in harmony with social norms and the process of subjective action and cognition after suffering from the PD. ${ }^{21}$ In the clinical practice of patients with $\mathrm{PD}$, it is not comprehensive to simply control the motor or non-motor symptoms of patients. ${ }^{22}$ Medical workers should also pay attention to the recovery of patients' psychosocial functions, improve the social adaptability and psychological adaptation level of patients with PD,

\begin{tabular}{lcccc}
\hline Groups & $n$ & $\begin{array}{c}\text { Total score of } \\
\text { stigma }\end{array}$ & $\begin{array}{c}\text { Internalized } \\
\text { stigma }\end{array}$ & $\begin{array}{c}\text { Enacted } \\
\text { stigma }\end{array}$ \\
\hline \hline $\begin{array}{l}\text { Family } \\
\text { function } \\
\text { was good }\end{array}$ & 132 & $51.87 \pm 10.54$ & $30.84 \pm 6.80$ & $21.03 \pm 4.77$ \\
$\begin{array}{l}\text { Family } \\
\text { dysfunction }\end{array}$ & 114 & $59.11 \pm 10.70$ & $35.44 \pm 6.92$ & $23.67 \pm 4.80$ \\
$t$ & & -5.329 & -5.243 & -4.311 \\
$P$ & & $<0.001$ & $<0.001$ & $<0.001$ \\
\hline
\end{tabular}

Table 2. Independent sample $t$-test of stigma in different family dysfunction groups.

\begin{tabular}{|c|c|c|c|c|c|c|c|c|}
\hline Groups & $n$ & $\begin{array}{c}\text { Total score of } \\
\text { psychosocial adaptation }\end{array}$ & Anxiety & $\begin{array}{l}\text { Self-esteem, } \\
\text { attitude }\end{array}$ & $\begin{array}{l}\text { Self-attitude } \\
\text { toward life }\end{array}$ & $\begin{array}{c}\text { Self- } \\
\text { acceptance }\end{array}$ & Self-efficacy & $\begin{array}{l}\text { Social } \\
\text { support }\end{array}$ \\
\hline $\begin{array}{l}\text { Family function } \\
\text { was good }\end{array}$ & 132 & $90.28 \pm 13.50$ & $18.97 \pm 3.68$ & $11.09 \pm 1.71$ & $16.54 \pm 2.42$ & $10.52 \pm 2.47$ & $13.18 \pm 1.93$ & $19.97 \pm 4.03$ \\
\hline $\begin{array}{l}\text { Family } \\
\text { dysfunction }\end{array}$ & 114 & $77.30 \pm 13.70$ & $16.11 \pm 3.42$ & $9.07 \pm 1.96$ & $13.65 \pm 3.00$ & $9.16 \pm 2.35$ & $11.51 \pm 2.07$ & $17.81 \pm 3.53$ \\
\hline$t$ & & 7.468 & 6.291 & 8.619 & 8.255 & 4.422 & 6.562 & 4.446 \\
\hline$P$ & & $<0.001$ & $<0.001$ & $<0.001$ & $<0.001$ & $<0.001$ & $<0.001$ & $<0.001$ \\
\hline
\end{tabular}

Table 3. Independent sample t-test of psychosocial adaptation in different family dysfunction groups. 
and promote the comprehensive recovery of patients. In the course of the patient's illness, family members and caregivers bear a huge burden which can lead to burnout since the caregivers make a significant contribution to people with PD that include economic, social, and personal losses. ${ }^{23}$ The results of this study proves that the better the family care, the better the psychosocial adaptation. The viewpoint of this research is basically consistent with Wu's. Wu et al. ${ }^{24}$ argued that a significant negative correlation existed between family function scores and the severity of depression and anxiety among potential donor candidates before liver transplantation. People with family dysfunction showed more depression and anxiety. In addition, Zhang ${ }^{25}$ found that there was a negative correlation between family care and depression in postpartum depression patients $(r=-0.638, P<0.01)$. More than that, Liu ${ }^{26}$ considered that perfect family function is the basis of social support system, an important external resource for patients to cope with diseases effectively, and an important part of patients' social adaptability. Influenced by 5000 years of Chinese culture, most Chinese believe that only by building a harmonious family can make the family member be happy. ${ }^{27}$ Long-term illness can cause patients to feel tired. The help of families can not only share the pressure of medical expenses but also play a supervisory role in patients' disease prediction, medication program,

\section{References}

1. Ma CL, SuL, Xie JJ, Long JX, Wu P, Gu L. The prevalence and incidence of Parkinson's disease in China: a systematic review and meta-analysis. J Neural Transm (Vienna). 2014;121:123-134.

2. Wang G, Cui HL, Liu J, et al. Progress of translational research on the pathogenesis, diagnosis and treatment of Parkinson's disease. Chin J Contemp Neurol Neurosurg. 2018;18:19-24 (in Chinese).

3. Wang LY, Liu LX, Lyu HJ, Fu DC. Research progress of anti - Parkinson's disease drugs. Chin Pharm. 2017;28:1143-1149 (in Chinese).

4. Jiang JL, Chen SY, Tsai ST. Quality of life in patients with Parkinson's disease after subthalamic stimulation: an observational cohort study for outcome prediction. Tzu Chi Med J. 2019;31:107-112.

5. Lu W. Study on Quality of Life of Patients with Parkinson's Disease and its Influencing Factors. Tayuan: Shanxi Medical University; 2015 (in Chinese).

6. Ma HI, Saint-Hilaire M, Thomas CA, Tickle-Degnen L. Stigma as a key determinant of health-related quality of life in Parkinson's disease. Qual Life Res. 2016;25:3037-3045. and exercise. It can improve patients' self-management and strengthen their confidence toward recovery. Therefore, the psychosocial adaptability of patients may be caused by family disharmony or family dysfunction. And the improvement of family function helps to improve the psychosocial adaptation of patients with PD. When solving the problem of patients' psychosocial adaptation, the medical staff can start their treatment process from the aspect of family care.

\section{Conclusions}

In summary, when patients with PD experience family conditions with increased functionality, stigma is reduced and psychosocial adaptation is improved. Strengthening the level of family care degree is an effective way to reduce stigma and improve patients' psychosocial adaptation. However, this study focuses only on one Three-A hospital in Dalian city and so the study on factors affecting stigma and psychosocial adaptation is not comprehensive. In the future, the sample size can be expanded to verify the results of this study.

\section{Conflicts of interest}

All contributing authors declare that there is no conflicts of interest.
7. Zhang $T$, Yin A, Sun X, Liu Q, Song G, Li L. Development, reliability and validity of the psychosocial adaptation scale for Parkinson's disease in Chinese population. Int J Clin Exp Med. 2015;8:19731-19738.

8. Parkinson's Disease and Movement Disorders Group, Neurology Branch, Chinese Medical Association. Diagnostic criteria for Parkinson's disease in China (2016 edition). Chin J Neurol. 2016;4:268271 (in Chinese).

9. Smilkstein G. The family APGAR: a proposal for a family function test and its use by physicians. J Fam Pract. 1978;6:1231-1239.

10. Lyu F, Zeng G, Liu SN, Zhong TL, Zhan ZQ. The reliability and validity of family care index questionnaire to measure family function in patients with cerebrovascular disease. Chin J Public Health. 1999;15: 987-988 (in Chinese).

11. Deng CY, Lu Q, Zhao Y. Development of the Chinese Version of Stigma Scale for Chronic Illness and test of its reliability and validity in stroke patients. Chin Gen Pract. 2017(34):4304-4309 (in Chinese). 
12. Min MM. Correlation of family care with self-burden and mental health in elderly patients with eye diseases. China J Health Psychol. 2018;26(12):17771780. (in Chinese)

13. Niu GF, Sun JP, Yang ZL, Song D, Sun RL. The correlation between family care and life satisfaction of the elderly in nursing institutions. Chin J Gerontol. 2018;38:6096-6098 (in Chinese).

14. Zhao $Y Y$, Wang $Y$, Lin $P$, Zhang $X M$. Family care status of 112 senile diabetic nephropathy patients with hemodialysis and its influencing factors. Chin Nurs Manag. 2016;16:488-490, 491 (in Chinese).

15. Sauerbier A, Jitkritsadakul O, Titova N, et al. Nonmotor symptoms assessed by non-motor symptoms questionnaire and non-motor symptoms scale in Parkinson's disease in selected Asian populations. Neuroepidemiology. 2017;49:1-17.

16. He YY, Liu SY, Huang XM, Yuan L, Yin AC. The research progress of stigma experience of people with Parkinson's disease. Med Phil. 2018;39:53-56 (in Chinese).

17. Maffoni M, Giardini A, Pierobon A, Ferrazzoli D, Frazzitta G. Stigma experienced by Parkinson's disease patients: a descriptive review of qualitative studies. Parkinson's Dis. 2017;2017:7203259.

18. Koch Filho HR, Koch LFA, Kusma SZ, et al. Selfperception of gerontoism according to social support and family functionality. Iran J Public Health. 2019;48:673-680.

19. Sun LN, Sun YH, Zhang DD, et al. Study on HIV related stigma and discrimination among people living with HIVIAIDS and their family members. Chin J Epidemiol. 2010;31:1219-1222 (in Chinese).
20. Zhang L, Yu R, Zheng QH, Yang Y. Study on relationship model between stigma, family function of breast cancer patients and their quality of life. Chin Nurs Res. 2017;31:1333-1336 (in Chinese).

21. Zhang TT. Development, Reliability and Validity of the Psychosocial Adaptation Scale for Parkinson's Disease. Dalian: Dalian Medical University; 2017 (in Chinese).

22. Shin JY, Habermann B. Nursing Research in Parkinson's disease from 2006 to 2015. Clin Nurs Res. 2017;26:142-156.

23. Mosley PE, Moodie R, Dissanayaka N. Caregiver burden in Parkinson disease: a critical review of recent literature. J Geriatr Psychiatry Neurol. 2017;30:235-252.

24. Wu MK, Hsu LW, Huang KT, et al. Assessment of relevant factors with respect to psychosocial properties in potential living donor candidates before liver transplantation. Neuropsychiatr Dis Treat. 2018;14:1999-2005.

25. Zhang JQ. Effects of family care on mental toughness and depression in postpartum depression patients. China Mod Med. 2018;25:136-139 (in Chinese).

26. Liu F. Survey of status quo of self-efficacy, social support and family care in inpatients with diabetes mellitus. Chin Nurs Res. 2015;29:4071-4073 (in Chinese).

27. Wang YW. The Family View of Engels and its Contemporary Inspiration [dissertation]. Chengdu: Sichuan Normal University; 2017 (in Chinese). 